\title{
A Prototype Head-Motion Monitoring System for In-Home Vestibular Rehabilitation Therapy
}

Pamela T. Bhatti ${ }^{1 \# *}$, Susan J. Herdman ${ }^{2}$, Siddarth Datta Roy ${ }^{1}$, Courtney D. Hall ${ }^{3,4}$ and Ronald J. Tusa ${ }^{5}$

${ }^{1}$ School of Electrical and Computer Engineering, Georgia Institute of Technology, Atlanta, GA, 30332-0250, USA

${ }^{2}$ Department of Rehabilitation Medicine, Emory University Atlanta, GA, 30322, USA

3 James H. Quillen VA Medical Center, Mountain Home, TN 37684, USA

${ }^{4}$ Department of Physical Therapy, East Tennessee State University, Johnson City, TN, 37614, USA

${ }^{5}$ Department of Neurology, Emory University, Atlanta, GA 30322, USA

${ }^{\#}$ P. Bhatti was supported in part by PHS Grant KL2 RR025009 from the Clinical and Translational Science Award program, National Institutes of Health, National Center for Research Resources

\begin{abstract}
This work reports the use of a head-motion monitoring system to record patient head movements while completing in-home exercises for vestibular rehabilitation therapy. Based upon a dual-axis gyroscope (yaw and pitch, \pm 500-degrees/sec maximum), angular head rotations were measured and stored via an on-board memory card. The system enabled the clinician to document exercises at home. Several measurements were recorded in one patient with unilateral vestibular hypofunction: The total time of exercise for the week (118 minutes) was documented and compared with expected weekly exercise time (140 minutes). For gaze stabilization exercises, execution time of 60 sec was expected, and observed times ranged from 75-100 sec. An absence of rest periods between each exercise instead of the recommended one minute rest period was observed. Maximum yaw head velocities from approximately 100-350 degrees/sec were detected. A second subject provided feedback concerning the ease of use of the HAMMS device. This pilot study demonstrates, for the first time, the capability to capture the head-motion "signature" of a patient while completing vestibular rehabilitation exercises in the home and to extract exercise regime parameters and monitor patient adherence. This emerging technology has the potential to greatly improve rehabilitation outcomes for individuals completing in-home gaze stabilization exercises ${ }^{1}$.
\end{abstract}

Keywords: Vestibular rehabilitation; Vestibular hypofunction; Gaze stabilization; Inertial sensors

\section{Introduction}

The vestibular system is responsible for maintaining gaze stability during head motion as well as maintaining postural stability while walking. Housed in the inner ear, the peripheral vestibular system consists of five end organs that detect head accelerations. Three (nearly) mutually orthogonal fluid-filled semicircular canals detect angular acceleration, and two otolith organs (utricle and saccule) detect linear acceleration. Each organ codes head motion as a neural firing rate and these signals are integrated in the central vestibular system with visual and proprioceptive information. An important reflex is mediated by the vestibular system- the angular vestibulo-ocular reflex (aVOR). The aVOR produces a compensatory eye movement to maintain a target image on the fovea of the retina as the head moves through space.

Dysfunction in the vestibular system can lead to dizziness, disorientation, decreased ability to walk, and blurred vision during head movement [1-3]. From 2001-2004, nearly $35.4 \%$ of adults in the US suffered from vestibular dysfunction [4]. When considering the impact that vestibular dysfunction has on an individual's quality of life, a major national study reports, "Difficulty in performing one or more activities of daily living (bathing, dressing, eating, getting in and out of bed, using toilet, getting around inside home) is highly prevalent among adults with balance problems (11.5\%) or dizziness $(33.4 \%)$ [5]." Furthermore, when considering dizziness alone, the incidence of dizziness increases with age, and $1.3 \%$ of individuals between the ages of 45-64 visit the internist for this reason [6]. Vestibular dysfunction and dizziness lead to falls $[7,8]$. Falls in the elderly are the leading cause

${ }^{1}$ The results of the first pilot study were briefly reported at the IEEE-AMA Conference in Boston, MA on Oct. 11, 2011. of fatal and non-fatal injuries for persons age $>65$ and a significant expense in healthcare at over \$23.3-billion dollars annually $[9,10]$.

Given the profound impact of normal gaze stability on balance confidence and reducing the incidence of falls, we focus our initial studies on critical gaze stabilization exercises. Such exercises foster the development of alternative strategies to improve gaze stability and also act to decrease a patient's perception of dizziness during head movements [11]. In patients with unilateral vestibular hypofunction completing such exercises, $80 \%$ improve [12-17]; however, there remain a number of patients who achieve no recovery or only a partial recovery. Lack of, or incomplete, recovery has a significant impact upon the quality of life for vestibular patients $[18,19]$. Thus, given the high rate of success with standard gaze stabilization exercises, there is an immediate need to pursue intensive treatment methods utilizing the combined disciplines of engineering and rehabilitation medicine to reach the $\sim 20 \%$ of patients who fail to improve [20].

In an ideal situation, a patient completing gaze stabilization exercises is supervised by a physical therapist (PT) during all phases

*Corresponding author: Pamela T. Bhatti, Assistant Professor, Department of Bioengineering, and Microelectronics/Microsystems, School of Electrical and Computer Engineering at the Georgia Institute of Technology, USA, Tel: 404.385.3144; E-mail: pamela.bhatti@ece.gatech.edu

Received December 05, 2011; Accepted January 06, 2012; Published January 11, 2012

Citation: Bhatti PT, Herdman SJ, Roy SD, Hall CD,Tusa RJ (2012) A Prototype Head-Motion Monitoring System for In-Home Vestibular Rehabilitation Therapy. J Bioengineer \& Biomedical Sci S1:009. doi:10.4172/2155-9538.S1-009

Copyright: ( 2012 Bhatti PT, et al. This is an open-access article distributed unde the terms of the Creative Commons Attribution License, which permits unrestricted use, distribution, and reproduction in any medium, provided the original author and source are credited. 
of exercise execution to ensure that the patient performs the exercises at the target head rotation rate, frequency, duration, and intervals throughout the day. While this may be feasible in a research setting, this is not realistic for contemporary health care practices where a PT may see a patient only once a week in the clinic and the patient is instructed to perform exercises on a daily basis at home. In the clinic, the PT guides the patient through the gaze stabilization exercises, modifies the exercises and verifies proper execution. The in-home exercises are designed to provide a greater intensity of therapy than would 1-3 visits a week to a PT clinic and, in our clinic, are performed four to five times daily for a total exercise time of up to 40 minutes $[16,17,21]$. Importantly, in-home exercises are unsupervised and patient self-reports is the only mode of verification of adherence with in-home exercises. Critical barriers to receiving the desired therapeutic benefit are: 1) incomplete participation in the prescribed therapeutic routine, and/or 2) sub-optimal execution of the exercises at home. Presently, there is no cost-effective way to measure and adjust these parameters outside of the clinic. To overcome these limitations, we propose the first Head Angular Motion-Monitoring System (HAMMS) for in-home exercise monitoring and eventual real-time performance feedback (Figure 1)

\section{Materials and Methods}

In this section we describe the system components of Head Angular Motion-Monitoring System (HAMMS) and a custom user interface to view subject data. This is followed by a description of the in-home exercise strategy followed by patients.

\section{System description}

A prototype head-worn system capable of capturing and storing human head rotation in both the yaw and pitch planes has been developed (Figure 2). Head rotations were measured with a dualaxis gyroscope housed on a third-party break out board (Sparkfun Electronics, Boulder, CO). The maximum detection capability of the gyroscope was \pm 500 degrees/sec in each rotation plane. Analog voltages (0.35-2.35 Volts) output from the gyroscope relaying angular head rotations were sampled at a rate of $100 \mathrm{~Hz}$ and converted into digital

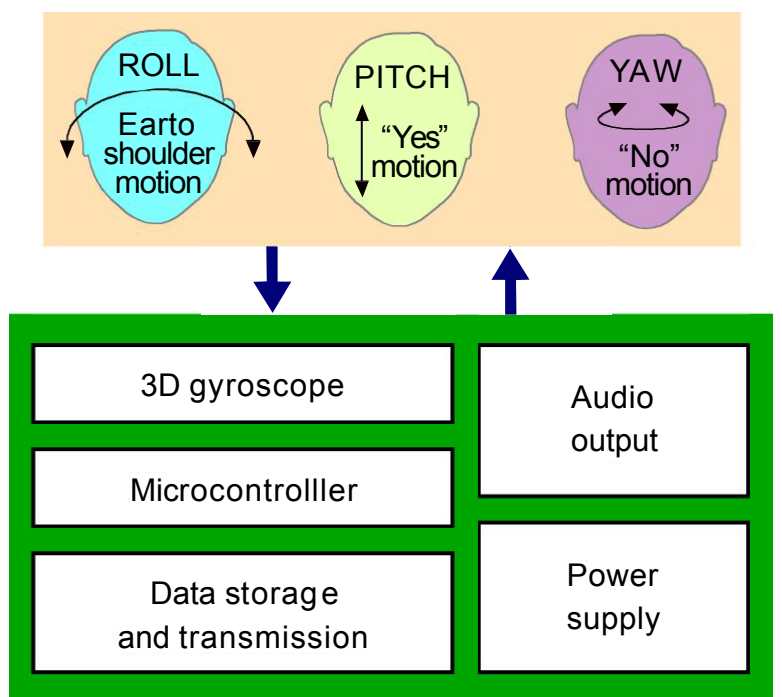

Figure 1: Block diagram of the Head Angular Motion-Monitoring System. A 3D gyroscope is shown for future sensing in all motion planes. In a future system audio feedback will be integrated to indicate to the patient when their head rotation is outside of clinically instructed range.

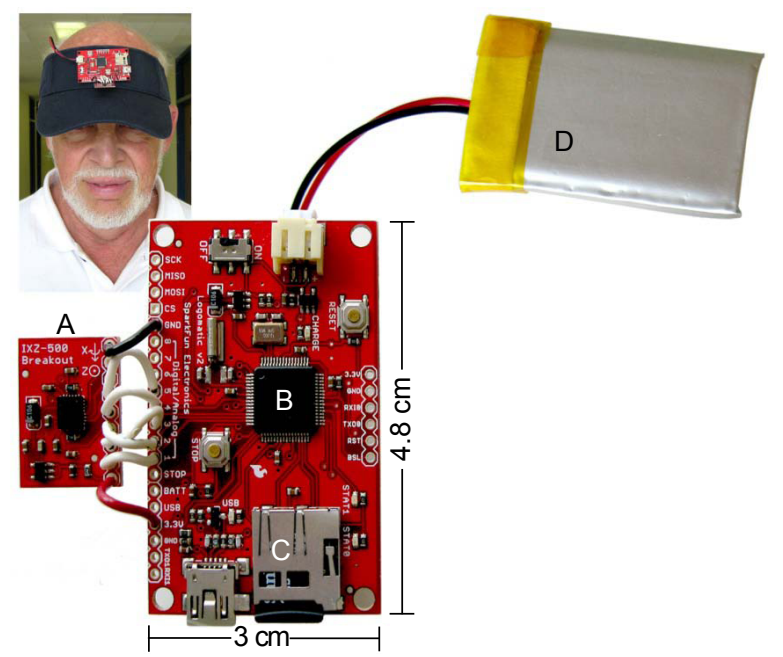

A. Dual axis gyro breakout board

B. Programmable Interface Controller

C. 1-Gbyte miniSD card

D. $900 \mathrm{mAh}$ Lithium Ion Battery

As shown, the visor limits the field of view. This was done to preserve the subject's privacy and is not representative of true placement during exercises.

Figure 2: Head Motion Monitoring System Components.

data with a 10-bit analog to digital convertor. Data was stored on a onegigabyte micro SanDisk flash memory card and downloaded through a Universal Serial Bus port. Components of the system were controlled through a programmable interface microcontroller and powered with a $900 \mathrm{mAh}$ Lithium-Ion battery. Given the battery life, the system is capable of monitoring motion for up to 22 days (11- hours total). To validate system operation, velocity measures were calibrated using a rate table (angular velocities ranging from 30 degrees /min to 1080 degrees $/ \mathrm{min}$ ) as well as in a rotary chair (60 degrees/sec and 240 degrees/sec) (Micromedical Technologies; Chatham, IL). To view and process the data, a custom MATLAB (MathWorks; Natick, MA) graphical user interface displayed yaw plane head motion velocity (Figure 3).

\section{Rehabilitation strategy}

Two types of gaze stabilization exercises are prescribed for the patient: adaptation and substitution [16]. Vestibular adaptation exercises are designed to improve gaze stability through long-term changes in the gain of the remaining vestibular system in response to input or the development of alternative strategies. Such exercises require the individual to fixate on a visual target during either horizontal or vertical head movement, which are performed for one minute at a time (Figure 4). Substitution exercises are designed to foster the use of alternative oculomotor strategies in order to substitute for lost vestibular function and maintain visual fixation. The specific substitution exercises used required the individual to perform eye-head movements between targets with the goal of seeing clearly during the head movement and were performed for one minute at a time.

In our clinic a patient is asked to perform the gaze stabilization exercises 4 to 5 times daily for a total of up to 40 minutes per day plus 20 to 30 minutes of balance and gait exercises per day. The change in exercises from week to week is a conservative progression in that it is designed to increase the patient's ability to maintain gaze stability and postural stability during head movement without increasing the patient's symptoms to a point where the symptoms interfere with 


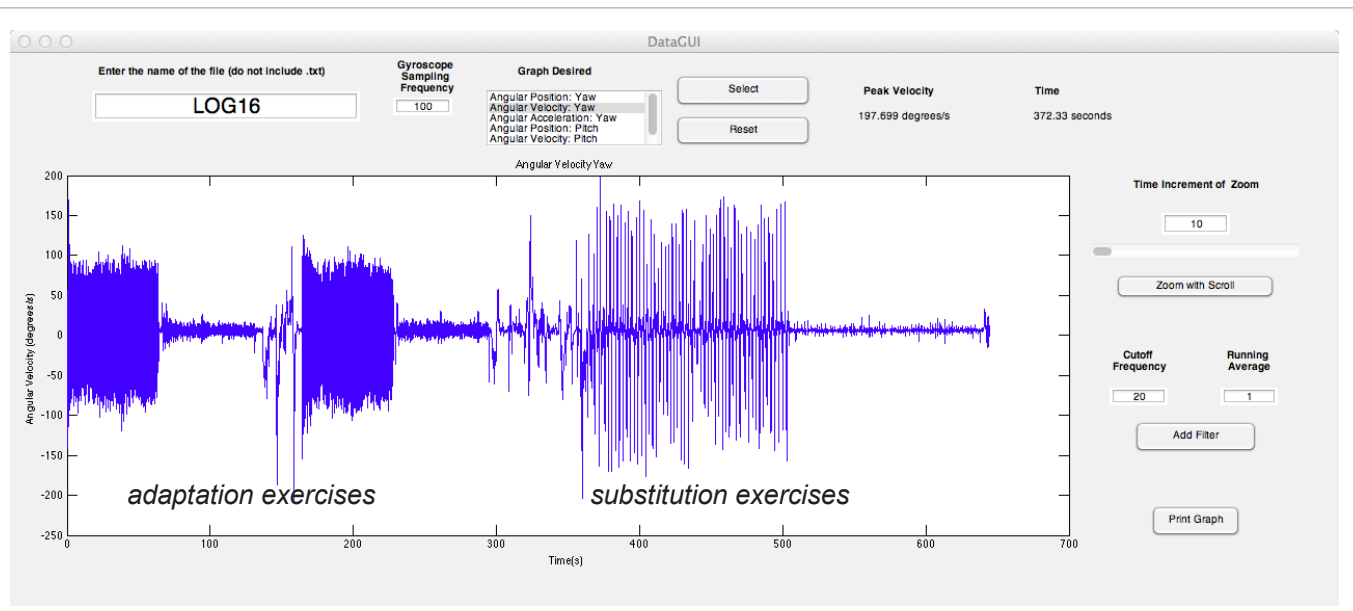

Figure 3: Graphical User Interface (GUI). A complete exercise session is shown for the yaw plane. The clinician may select to view the angle, velocity, or angular acceleration during head rotations in either the yaw or pitch plane. The clinician may also examine rest phases by adjusting the time epoch. A low pass filter cutoff frequency may be specified to further process the data by removing low frequency noise. Two types of exercise signatures are present in the session. The first, adaptation exercises, required the individual to fixate on a visual target during either horizontal or vertical head movement, which are performed for one minute at a time. The substitution exercises required the individual to perform eye-head movements between targets with the goal of seeing clearly during those tasks.
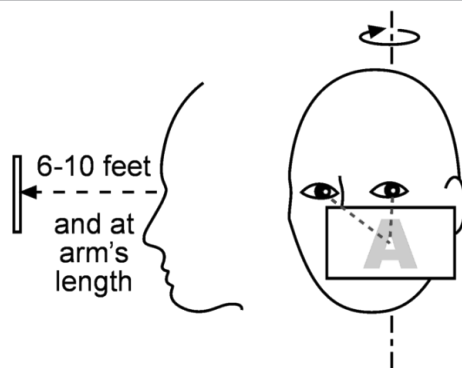
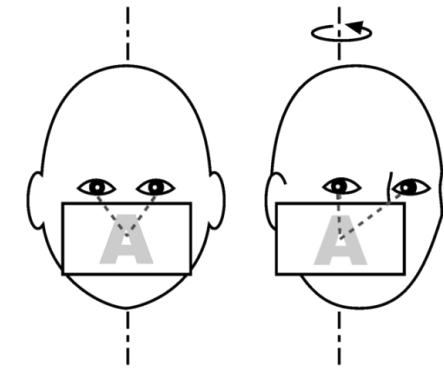

Figure 4: Cartoon of gaze stabilization exercises in the yaw plane. Adaptation exercises require the individual to fixate on a visual target (indicated by the letter ' $A$ ') during either horizontal or vertical head movement, which are performed for one minute at a time. The individual first completes the exercises with a target affixed to a wall 6-10 feet away. During the second exercise, the individual holds the target at eye level. These vestibular adaptation exercises are designed to improve gaze stability through long-term changes in the gain of the remaining vestibular system in response to input or the development of alternative strategies.

the patient's daily activities. Patients are asked to follow the program unless they reported difficulties related to increases in their complaints of dizziness. If the exercises exacerbate the dizziness, the patient is instructed to work through the dizziness if possible. If this is not possible, the first step is to modify the exercises by decreasing the head velocity to a more tolerable level. If this does not relieve symptoms, the frequency of performing the exercises will be reduced from 5 times daily to 3 times daily for 3 days, then, if possible, the frequency of performance is again increased to five times daily $[16,17]$. Patients are seen weekly for review of their progress and revision of the exercise program as needed. All patients record adherence with eye exercises on a weekly calendar and verbally report adherence with balance and gait exercises. An adherence measure is estimated from weekly calendar reports among patients visiting the outpatient center and typically ranges from $50-75 \%[16]$.

\section{Results}

Two subjects have tested a pilot system ${ }^{2}$. The first subject, a patient from Emory Dizziness and Balance Center (Emory University Hospital, Atlanta, GA) wore the HAMMS during all in-home exercises during the second week of the exercise regime. The patient was a 52 year-old male with a resection of a left acoustic neuroma leading to unilateral ${ }^{2}$ Informed consent was obtained in compliance with institutional review board protocol of Emory University, Atlanta, GA vestibular loss. The patient was post radiosurgery for vestibular schwanoma and was minimally hearing impaired on the left only. During the clinical visit the patient was taught the exercises and given written instructions with illustrations on how to perform the exercises. The PT also taught the patient how to position the HAMMS cap on his head. Within 10-minutes the patient reached a skill level necessary for safe and effective use of the device. This particular individual displayed a basic level of understanding of typical consumer electronics (e.g. cell phone) and was high school educated. He was instructed to perform the gaze stabilization exercises four times daily while wearing the HAMMS. The patient was also instructed to complete balance and gait exercises totaling 20 minutes daily. At the following clinical visit, the patient's data was downloaded to a personal computer using the memory card embedded in the HAMMS visor. This procedure was similar to transferring images from a digital camera memory card onto a personal computer. To view and process the data, the graphical user interface was employed. Execution parameters including maximum head velocity, exercise type (adaptation vs. substitution), and rest periods were determined. A representative sample of one exercise session recorded by the HAMMS is illustrated in Table 1. Figure 3 depicts the total 644 seconds for the session and Figure 5 illustrates the initial segment of the session. Using the complete record from the HAMMS a total time of exercise for the week (118 minutes) was computed and compared with expected weekly exercise time (140 minutes). For adaptation exercises, 
Citation: Bhatti PT, Herdman SJ, Roy SD, Hall CD,Tusa RJ (2012) A Prototype Head-Motion Monitoring System for In-Home Vestibular Rehabilitation Therapy. J Bioengineer \& Biomedical Sci S1:009. doi:10.4172/2155-9538.S1-009
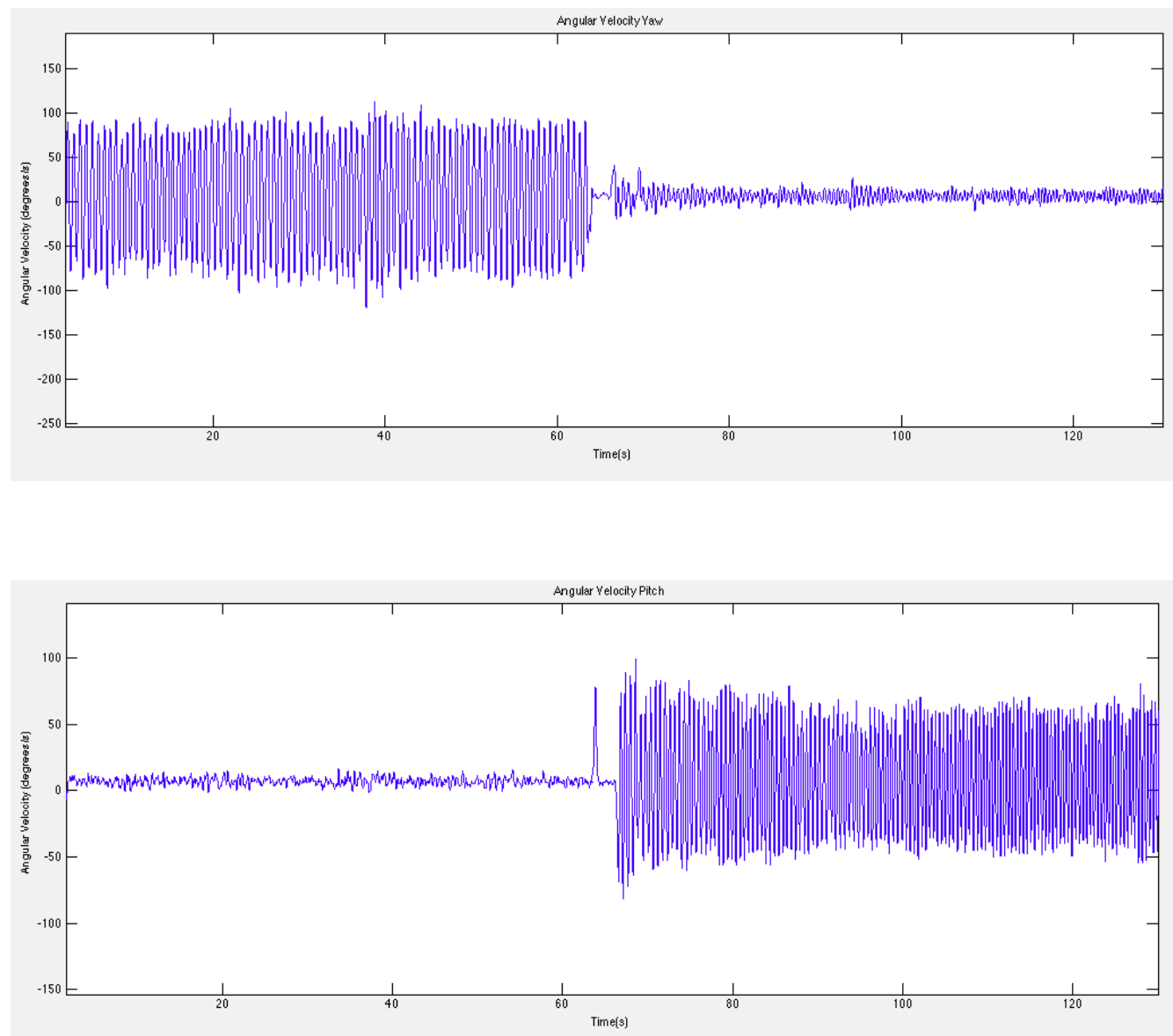

Figure 5: Raw data from a captured exercise session. Head rotation in the yaw plane (top) and pitch plane (bottom) are shown. As suggested the patient completed approximately one minute of gaze stabilization (adaptation) exercises in each plane. It is important to note that while the patient was instructed to rest for one minute between exercises in each plane, the data indicates a rest period of approximately $4 \mathrm{sec}$. The PT notes that the patient required re-education on exercise execution so the patient would include the one minute rest periods. The one minute rest period is recommended because prolonged head movements may heighten the intensity and duration of dizziness and cause the patient to avoid performing the exercises or limit other activities. Off-axis signals are also observed due to the difference in alignment between the gyroscope planes and head rotations planes.

\begin{tabular}{|c|c|c|c|c|c|c|}
\hline \multicolumn{7}{|c|}{ HAMMS LOG 16: 10 minutes, 44 seconds } \\
\hline \multicolumn{6}{|c|}{ Segment estimates } & \multirow[t]{2}{*}{ Clinical comments } \\
\hline Exercise type & Head rotation & $\begin{array}{l}\text { Instructed duration } \\
(\mathrm{sec})\end{array}$ & $\begin{array}{l}\text { Recorded duration } \\
(\mathrm{sec})\end{array}$ & $\begin{array}{l}\text { Start to stop time } \\
(\mathrm{sec})\end{array}$ & $\begin{array}{l}\text { Maximum recorded } \\
\text { amplitude } \\
\text { (degrees/sec) }\end{array}$ & \\
\hline \multirow[t]{8}{*}{ Adaptation } & yaw & 60 & 64 & $0-64$ & 100 & \multirow{12}{*}{$\begin{array}{l}\text { Based on the data abstracted from the } \\
\text { HAMMS LOG, the patient waited only } 0-20 \\
\text { seconds between each set of exercises see } \\
\text { rest periods to left) instead of the prescribed } \\
60 \text { seconds. Based on this information, the } \\
\text { patient was re-educated on appropriate du- } \\
\text { ration of the rest periods }\end{array}$} \\
\hline & rest & 60 & 4 & $64-68$ & - & \\
\hline & pitch & 60 & 62 & $68-130$ & 60 & \\
\hline & rest & 60 & 20 & $130-150$ & - & \\
\hline & yaw & 60 & 78 & $150-228$ & 110 & \\
\hline & rest & 60 & 10 & $228-238$ & - & \\
\hline & pitch & 60 & 56 & $238-294$ & 80 & \\
\hline & rest & as needed & 66 & $294-360$ & - & \\
\hline \multirow[t]{4}{*}{ Substitution } & yaw & 120 & 144 & $360-504$ & 150 & \\
\hline & rest & as needed & 0 & - & - & \\
\hline & pitch & 120 & 132 & $504-636$ & 110 & \\
\hline & \multicolumn{5}{|c|}{ Rest briefly before gait exercises } & \\
\hline
\end{tabular}

Table 1: Sample of captured exercise session.

execution times of $60 \mathrm{sec}$ were expected while observed times ranged from 75-100 sec. For substitution exercises, execution times of $120 \mathrm{sec}$ were expected while observed times ranged from 100-140 sec. During exercises maximum yaw head velocities ranged from approximately 100-350 degrees/sec and maximum pitch velocities ranged from 60140 degrees/sec. No rest periods were noted between each exercise. 
The second subject tested a revised version of the system where the circuitry was moved to the inside lining of the visor to both protect the circuitry and to reduce the size of the device for cosmetic reasons. This subject was a healthcare provider with a graduate degree. Functionally, the processor board was upgraded (Arduino Pro Mini; Sparkfun Electronics) to enable integration of a clock to timestamp each exercise session, wireless data transfer via a Bluetooth interface, and eventual real-time auditory feedback control.

\section{Discussion}

We have prototyped a compact system that can be used to monitor head motion in patients performing vestibular rehabilitation exercises at home. Using this prototype, we conducted a pilot study that demonstrated for the first time the capability to capture the motion "signature" of a patient while completing gaze stabilization exercises in the home. By extracting exercise regime parameters, a PT is able to make essential performance observations. As indicated by the session $\log$ in Table 1, although the patient was completing gaze stabilization exercises as instructed, he failed to introduce a necessary break between exercises. The PT can potentially correlate this information if a patient reports greatly increased dizziness during exercises. At a minimum, the PT can use these data to determine that the patient requires reeducation about the exercises. When examining the last session log of the week (not shown), the PT observed an increase in head velocity during the eye-head substitution exercise potentially indicating that the patient was improving. In summary, the observation of such measures is critical in developing patient-specific exercise regimes to improve outcome and decrease therapy duration.

From the standpoint of HAMMS usability, each subject felt that the system did not interfere with exercise execution and anecdotally commented that wearing the visor served to place him/her in the context of an exercise session. Although the battery remained charged for the duration of a week, both subjects suggested that turning the system on and remembering to turn it off was cumbersome and a timeout feature has now been integrated.

Our results demonstrate an important first-step toward an inexpensive ( $\sim 100$ US dollars), low-power, user-friendly technique for tracking and optimizing rehabilitation exercises. More specifically, the HAMMS could have a significant impact on improving adherence by both monitoring and guiding patients through in-home rehabilitation exercises. Recent investigation on vestibular motor adaptation indicates that for individuals with an abnormally low vestibulo-ocular reflex gain, training with progressively more retinal slip errors is more effective at increasing the gain than with large sudden slip errors [22]. These results underscore the need for a dynamic, programmable in-home system that enables exercise progression. In addition the HAMMS will lead to improved monitoring and standardization of gaze stabilization exercises. A PT has a very limited sample of patient performance during a one-hour visit and must use this to plan the course of treatment. There is considerable variability in the treatment planning for vestibular rehabilitation among clinics and even within a single clinic. The HAMMS and associated usage protocol has the potential to radically standardize treatment planning both within and among rehabilitative clinics.

The HAMMS has the potential to improve clinical outcomes as well as reduce the duration of treatment by providing the PT with information about exercise performance and compliance. A patient could also use the HAMMS once formal PT has ended in order to provide the clinician with simple diagnostic tool of gaze stabilization without a complete clinical visit. For example, a patient could complete a series of gaze stabilization exercises in-home and upload the data to a secure server. This could be achieved through a wired link or a wireless link. In the current design, a memory card resides in the visor and the patient could transfer the data to a computer and upload it to the server. Currently we are developing a wireless interface for real-time data transmission to a smart phone and ultimately to a secure server. On the clinical side, a PT could then access patient data and compares these results (e.g. maximum velocity) with the discharge measures and flag a potential loss in function. By more tightly coupling the patientclinic loop, follow-up interventions can occur earlier and potentially reduce duration of follow-up therapy.

\section{Acknowledgement}

This work was supported in part by National Science Foundation BRIGE Award 0927103 and by PHS Grant KL2 RR025009 from the Clinical and Translational Science Award program, National Institutes of Health, National Center for Research Resources. The authors thank Wanchao Gao, Andrew Durand and James Steinberg from the School of Electrical and Computer and Engineering at the Georgia Institute of Technology for hardware development, as well as Diana Fouts for graphical support. The authors also thank Charles Bering, AuD and Lisa Heusel-Gillig, DPT from the Emory Dizziness and Balance Center for testing assistance.

\section{References}

1. Gillespie MB, Minor LB (1999) Prognosis in bilateral vestibular hypofunction Laryngoscope 109: 35-41.

2. Grunbauer WM, Dieterich M, Brandt T (1998) Bilateral vestibular failure impairs visual motion perception even with the head still. Neuroreport 9: 1807-1810.

3. Minor LB (1998) Gentamicin-induced bilateral vestibular hypofunction. JAMA 279: $541-544$

4. Agrawal Y, Carey JP, Della Santina CC, Schubert MC, Minor LB (2009) Disorders of balance and vestibular function in US adults: data from the National Health and Nutrition Examination Survey, 2001-2004. Arch Intern Med 169: 938-944.

5. Ko C, Hoffman HJ, Sklare DA (2006) Chronic imbalance or dizziness and falling: results from the 1994 disability supplement to the national health interview survey and the second supplement on aging study. Press at the Annual Meeting of the Association for Research in Otolaryngology, Baltimore.

6. Herdman SJ (2007) Management of the elderly person with vestibular hypofunction. Vestibular Rehabilitation. (3rdedn) F.A. Davis Company, Philadelphia

7. Herdman SJ, Blatt P, Schubert MC, Tusa RJ (2000) Falls in patients with vestibular deficits. Am J Otol 21: 847-851.

8. Whitney SL, Marchetti GF, Schade Al (2006) The relationship between falls history and computerized dynamic posturography in persons with balance and vestibular disorders. Arch Phys Med Rehabil 87: 402-407.

9. Centers for Disease Control and Prevention (CDC) (2008) Self-reported falls and fall-related injuries among persons aged $>$ or $=65$ years--United States, 2006. MMWR Morb Mortal Wkly Rep 57: 225-229.

10. Davis JC, Robertson MC, Ashe MC, Liu-Ambrose T, Khan KM, et al. (2010) International comparison of cost of falls in older adults living in the community: a systematic review. Osteoporos Int 21: 1295-1306.

11. Schubert MC, Hall CD, Das V, Tusa RJ, Herdman SJ (2010) Oculomoto strategies and their effect on reducing gaze position error. Otol Neurotol 31 228-231.

12. Badke MB, Shea TA, Miedaner JA, Grove CR (2004) Outcomes after rehabilitation for adults with balance dysfunction. Arch Phys Med Rehabil 85 227-233.

13. Cohen HS, Kimball KT (2003) Increased independence and decreased vertigo after vestibular rehabilitation. Otolaryngol Head Neck Surg 128: 60-70.

14. Enticott JC, O'leary SJ, Briggs RJ (2005) Effects of vestibulo-ocular reflex exercises on vestibular compensation after vestibular schwannoma surgery. Otol Neurotol 26: 265-269. 
Citation: Bhatti PT, Herdman SJ, Roy SD, Hall CD,Tusa RJ (2012) A Prototype Head-Motion Monitoring System for In-Home Vestibular Rehabilitation Therapy. J Bioengineer \& Biomedical Sci S1:009. doi:10.4172/2155-9538.S1-009

15. Hall CD, Heusel-Gillig L, Tusa RJ, Herdman SJ (2010) Efficacy of gaze stability exercises in older adults with dizziness. J Neurol Phys Ther 34: 64-69.

16. Herdman SJ, Schubert MC, Das VE, Tusa RJ (2003) Recovery of dynamic visual acuity in unilateral vestibular hypofunction. Arch Otolaryngol Head Neck Surg 129: 819-824

17. Herdman SJ, Hall CD, Schubert MC, Das VE, Tusa RJ (2007) Recovery of dynamic visual acuity in bilateral vestibular hypofunction. Arch Otolaryngol Head Neck Surg 133: 383-389.

18. Cohen HS, Wells J, Kimball KT, Owsley C (2003) Driving disability and dizziness. J Safety Res 34: 361-369.
19. Viciana D, Lopez-Escamez JA (2010) Vestibular evoked myogenic potentials and health-related quality of life in patients with vestibular neuritis. Oto Neurotol 31: 954-958.

20. Herdman SJ, Hall CD, Delaune W (2011) Variables Associated With Outcome in Patients With Unilateral Vestibular Hypofunction. Neurorehabil Neural Repair.

21. Hall CD, Schubert MC, Herdman SJ (2004) Prediction of fall risk reduction as measured by dynamic gait index in individuals with unilateral vestibular hypofunction. Otol Neurotol 25: 746-751.

22. Schubert MC, Zee DS (2010) Saccade and vestibular ocular motor adaptation Restor Neurol Neurosci 28: 9-18. 